\title{
A Low-Cost and Portable Smart Instrumentation for Detecting Colorectal Cancer Cells
}

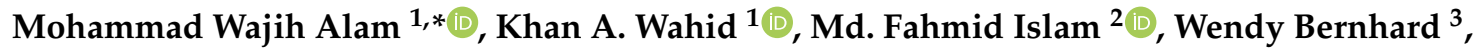 \\ Clarence R. Geyer ${ }^{3}$ and Franco J. Vizeacoumar ${ }^{2}$ \\ 1 Department of Electrical and Computer Engineering, University of Saskatchewan, Saskatoon, Saskatchewan, \\ SK S7N 5A9, Canada \\ 2 Saskatchewan Cancer Agency, Division of Oncology, University of Saskatchewan, Saskatoon, Saskatchewan, \\ SK S7N 5E5, Canada \\ 3 Department of Pathology and Laboratory Medicine, University of Saskatchewan, Saskatoon, Saskatchewan, \\ SK S7N 0W8, Canada \\ * Correspondence: wajih.alam@usask.ca
}

Received: 21 July 2019; Accepted: 14 August 2019; Published: 26 August 2019

check for updates

\begin{abstract}
Fluorescence imaging is a well-known method for monitoring fluorescence emitted from the subject of interest and provides important insights about cell dynamics and molecules in mammalian cells. Currently, many solutions exist for measuring fluorescence, but the application methods are complex and the costs are high. This paper describes the design and development of a low-cost, smart and portable fluorimeter for the detection of colorectal cancer cell expressing IRFP702. A flashlight is used as a light source, which emits light in the visible range and acts as an excitation source, while a photodiode is used as a detector. It also uses a longpass filter to only allow the wavelength of interest to pass from the cultured cell. It eliminates the need of both the dichroic mirror and excitation filter, which makes the developed device low cost, compact and portable as well as lightweight. The custom-built sample chamber is black in color to minimize interference and is printed with a $3 \mathrm{D}$ printer to accommodate the detector circuitry. An established colorectal cancer cell line (human colorectal carcinoma (HCT116)) was cultured in the laboratory environment. A near-infrared fluorescent protein IRFP702 was expressed in the colorectal cancer cells that were used to test the proof-of-concept. The fluorescent cancer cells were first tested with a commercial imaging system (Odyssey ${ }^{\circledR} \mathrm{CLx}$ ) and then with the developed prototype to validate the result in a preclinical setting. The developed fluorimeter is versatile as it can also be used to detect multiple types of cancer cells by simply replacing the filters based on the fluorophore.
\end{abstract}

Keywords: fluorescence imaging; fluorimeter; colorectal cancer; IRFP702; fluorescence microscopy

\section{Introduction}

The combined advancement in the field of molecular biology and optics has revolutionized the investigation methods used in the field of biology and chemistry. The progress in fluorescence microscopy and molecular tagging has also enabled the pathways to analyze the dynamics of cells with molecule-specific contrast [1,2]. In recent years, these fluorescent microscopes have garnered a lot of attention, as they offer multiple benefits and possibilities including live-cell imaging, high-resolution images and localization of fluorescence in cells. However, these fluorescent microscopes are expensive as they use sensitive and sophisticated equipment/parts [3] making it affordable only to hospitals/institutions with sound financial resources. These microscopes also require a trained operator [4] in order to utilize their functionality efficiently, such as adjusting the illumination, optical alignment, correct lens changeovers, filtration techniques and capturing images. A few low-cost 
fluorescent microscopes were developed recently [5-7] to make it more affordable to researchers from developing countries but the low resolution still obstructs its wide application. On the other hand, fluorimeters measure fluorescent signal intensity emitted from the dyes that are attached to the biological molecules as well as naturally fluorescent molecules based on their excitation and emission wavelengths. These devices are commonly used for monitoring chlorophyll [8-10] in water. Monitoring these fluorescent signal helps researchers to find out the presence of specific molecules in the targets of interest [11] based on signal intensity [12]. A recent work [12] showed the detection of breast cancer cells with the help of a fluorometer. However, these devices are often bulky and costly as well as vulnerable to inconsistent fluctuation caused due to the changing environment, sensors and instrument design, as well as calibration of the equipment/parts.

Colorectal cancer is the third most common type of cancer diagnosed in both men and women. It is also the third most leading cause of cancer-related deaths in the United States [13]. However, diagnosing cancer requires the use of expensive equipment (such as magnetic resonance imaging (MRI)) as well as a trained operator, which limits its use in the developing countries. Furthermore, in many regions around the world, the resources are limited due to the high cost of instrumentation as well as lab supplies. Though colorectal cancer mortality rates have declined mainly due to the advancement in technology, early and effective screening is needed to further reduce associated mortalities. The most common clinical procedure employed for diagnosing cancer includes colonoscopy (the gold standard) [14] which is both invasive and relatively expensive. This procedure is complex and is accompanied by high costs of operation. Providing each individual with hands-on access to commercial equipment requires either multi-week training through a set of experiments or the deployment of multiple instruments, which therefore has been both cost and time prohibitive.

The use of fluorescence microscopy to visualize living cells is often achieved via specific labelling of proteins [15] through antibody, bio-specific ligand or nanobody that is conjugated to a fluorophore. Fluorescent labelling is usually achieved by using a reactive derivative of the fluorophore, which specifically binds to a selective group contained in the target molecule. Several studies have shown the detection of cancer cells by labelling the proteins of interest. In association with the recent development of biomarker sciences, fluorescence imaging is being used as a tool to detect complex human diseases based on targetable biomarkers. For example, cancer cells express many biomarkers which can be utilized to develop fluorescent probes (e.g., fluorescent antibodies) and detect cancer tissues with fluorescent microscopy or imaging instruments $[16,17]$. Although these techniques utilize ultrasensitive imaging instruments for monitoring the fluorescent signal, these modern systems are costly and need dedicated software as well as sophisticated hardware. Hence, it is a need that a low-cost device is developed which can help to differentiate between the fluorescent cancer cells and control cells (colorectal cancer cell which does not express IRFP702 fluorophore). In this work, we designed and developed a portable fluorimeter utilizing off-the-shelf components which is not only low-cost but also sensitive enough to distinguish between the IRFP702 conjugated cancer cells and control cells.

\section{Materials and Methods}

Most substances absorb light. The substances which absorb light at a particular wavelength and energy but emit light at a longer wavelength but lower energy are called fluorescence substances and the principle is called fluorescence. Fluorescent dyes or quantum dots are most commonly used as fluorescence substance. Hence, when a light of sufficient intensity is incident upon samples which are conjugated with a fluorophore dye, these samples absorb light and emit at a longer wavelength. However, these are of low intensity. The proposed device exploits this principle of fluorescence to detect colorectal cancer cells. Figure 1a shows the working principle of a typical fluorescence microscope [7], whereas Figure $1 b$ shows the working principle of the proposed fluorimeter. In this paper, IRFP702 is used as a dye which is conjugated with colorectal cancer cells (human colorectal carcinoma (HCT116)). The peak absorption and emission wavelength of infrared protein (IRFP702) are $673 \mathrm{~nm}$ and $702 \mathrm{~nm}$ respectively. As can be seen in Figure 1b, the excitation light (in the visible range) from the flashlight 
is focused on the top of the sample. The emitted light at a longer wavelength from the sample is then passed through a hard-coated longpass filter (Edmund Optics, Stock\# 62-980) whose longpass wavelength is $700 \mathrm{~nm}$. The fluorescent light is detected by the photodiode. The photodiode is placed inside a custom-built sample chamber which is black in color to minimize the interference from the ambient light. The microcontroller reads the data from the photodiode and displays it on an liquid crystal display (LCD).

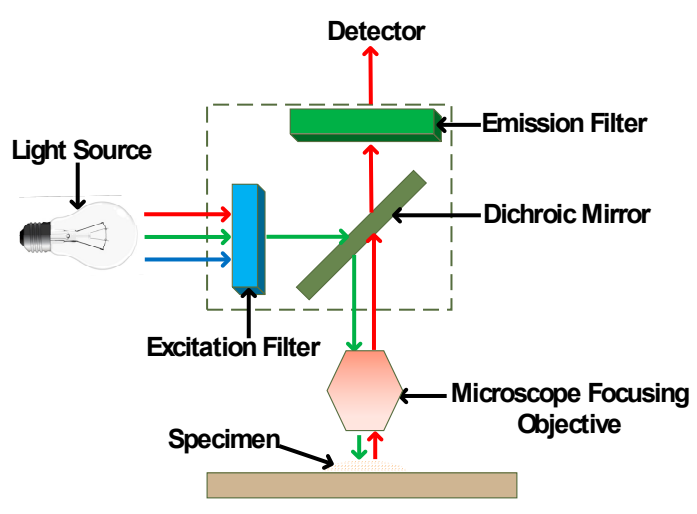

(a)

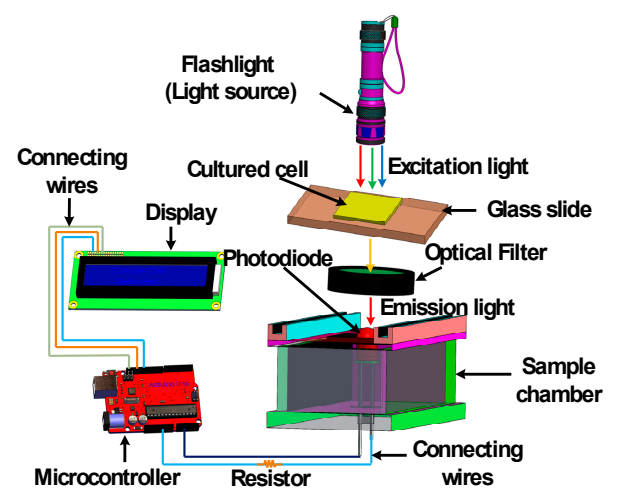

(b)

Figure 1. Fluorescence imaging: (a) working principle of a typical fluorescence microscope and (b) a three-dimensional illustration of the proposed fluorimeter.

The proposed device is comprised of a flashlight (Ultrafire C8) [18], a photodiode (OSRAM Opto Semiconductors SFH 203) [19,20], a microcontroller (Arduino UNO REV3 (surface-mount device) SMD version) [21] and a LCD (Basic $16 \times 2$ Character LCD—White on Black 3.3 V version) screen [22] as major components. The photodiode is used to detect fluorescent signal while the microcontroller is used to read and transmit data detected from the photodiode to the LCD display. The chosen photodiode has a short switching time of $5 \mathrm{~ns}$ while its responsivity lies in the range of 400-1100 $\mathrm{nm}$ and is available in a $5 \mathrm{~mm}$ LED plastic packaging. The relative spectral sensitivity of this photodiode at $702 \mathrm{~nm}$ is 0.82 and it has a radiant sensitive area of $1 \mathrm{~mm}^{2}$. This wide spectral characteristic of the photodiode makes it versatile and an ideal candidate to be used in the designed fluorimeter. The overall working principle is shown in Figure $1 \mathrm{~b}$ where it is clear that the flashlight is placed in a straight line on top of the sample. The sample is placed in between the longpass filter and flashlight. The optical longpass filter/high pass filter is designed to transmit wavelengths greater than the cut-on wavelength of the filter and block the lower wavelengths. The detector circuitry is placed inside the sample chamber. The received signal from the photodiode is processed with the help of an Arduino Uno microcontroller and displayed on an LCD display. The major components that are required to assemble the fluorimeter are shown in Figure 2. The chosen flashlight has a dimension of $14.5 \mathrm{~cm} \times 4.5 \mathrm{~cm} \times 4.5 \mathrm{~cm}$ and can operate in three modes: strobe, low and high. It is operated with a rechargeable 3.7 li-ion battery and has a $3000 \mathrm{mAh}$ current rating. For this experiment, the flashlight was operated in high mode. It uses a Cree XR-E Q5 type emitter, which has a luminous flux of $200 \mathrm{~lm}$ [23]. The sample chamber is designed with Solidworks ${ }^{T M}$ and printed with the help of a 3D printer (CR-10), which is black in color in order to reduce unwanted noise. The complete dimension of the custom-built sample chamber is shown in Figure 3. To further minimize the interference, a longpass filter is used to pass only the fluorescence wavelengths while filtering out the unwanted spectrum from the flashlight. This longpass filter is placed between the sample and the photodiode. The measured signal is then processed using the microcontroller. 


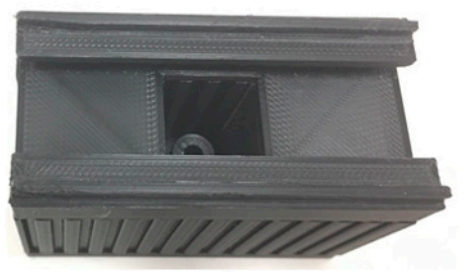

(a)

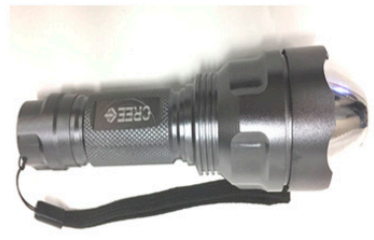

(d)

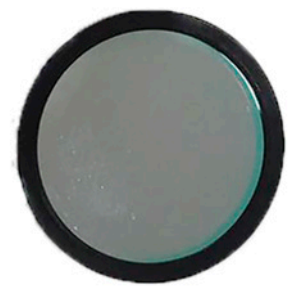

(b)

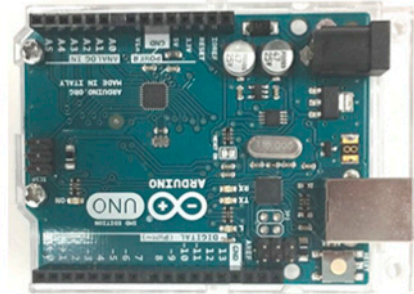

(c)

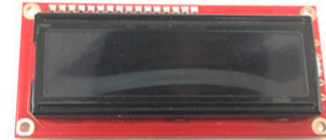

(f)

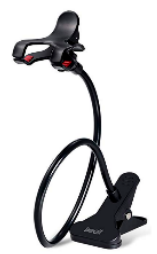

(g)

Figure 2. Off-the-shelf commercial components used to construct the prototype: (a) 3D printed sample chamber, (b) longpass filter, (c) microcontroller (Arduino Uno), (d) flashlight, (e) photodiode, (f) LCD display, and (g) holder (images are not to scale).

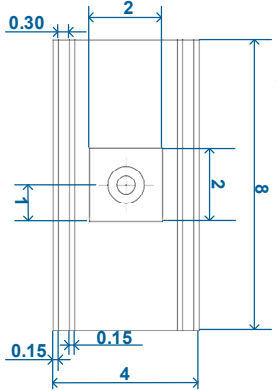

(a)

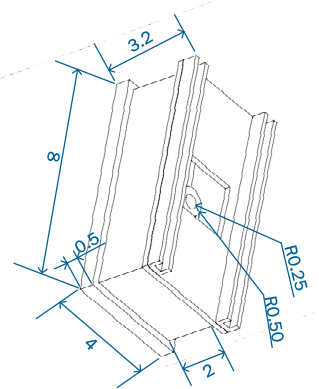

(b)

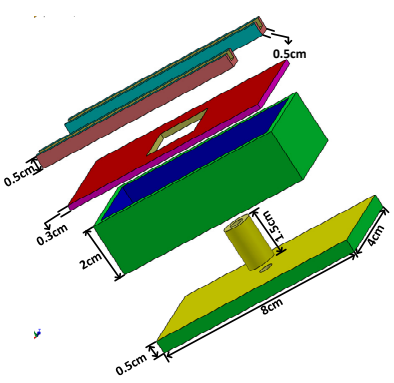

(c)

Figure 3. Dimension of custom-built sample chamber printed with 3D printer which houses detector circuitry (CR-10): (a) top view, (b) isometric view, and (c) 3-dimensional view (exploded). All dimensions in this figure are given in $\mathrm{cm}$.

IRFP702 fluorophore was expressed in the colorectal cancer cells (HCT116) using the lentiviral transduction method to test the developed prototype. The gene encoding IRFP702 was cloned into the pHAGE plasmid backbone (Addgene, Watertown, MA, USA) to construct pHAGE-IRFP702. To prepare a lentivirus, $8 \times 10^{5}$ 293-F (Thermo Fisher Scientific, Waltham, MA, USA) cells were plated for 24 $\mathrm{h}$ in $90 \%$ Dulbecco's Modified Eagle Medium (DMEM) medium (Thermo Fisher Scientific). One microgram of pHAGE-IRFP702 was mixed with $0.5 \mu \mathrm{g}$ of pSPAX.2 (Addgene) and $0.5 \mu \mathrm{g}$ pMD2.G (Addgene) in $100 \mu \mathrm{L}$ of Opti-Eagles minimum essential media (Opti-MEM) (Thermo Fisher Scientific). Polyethylenimine (PEI) (Sigma-Aldrich, Saint Louis, MO, USA) was added to $100 \mu \mathrm{L}$ of Opti-MEM to a final concentration of $120 \mu \mathrm{g} / \mathrm{ml}$. The DNA and PEI mixtures were mixed together and incubated at room temperature for $20 \mathrm{~min}$ to prepare a transfection mix. The transfection reaction was added to the cells drop-wise and incubated at $37^{\circ} \mathrm{C}$ with $5 \% \mathrm{CO}_{2}$. The virus was collected $72 \mathrm{~h}$ after the transfection. For the transduction, $1.5 \times 10^{5} \mathrm{HCT} 116$ cells (American Type Culture Collection) were plated for $24 \mathrm{~h}$ in HyClone ${ }^{\mathrm{TM}}$ McCoy's 5A Media (Fisher Scientific, Pittsburgh, PA, USA). The next day, the media was removed and replaced with $500 \mu \mathrm{L}$ of $90 \%$ McCoy's $5 \mathrm{~A}$ medium with $4 \mu \mathrm{g} / \mathrm{mL}$ of polybrene (Sigma-Aldrich) and incubated at $37^{\circ} \mathrm{C}$ for $10 \mathrm{~min}$. Five $\mu \mathrm{L}$ of the virus was added to the cells and then centrifuged at $1500 \times g$ for one hour. The cells were incubated at $37^{\circ} \mathrm{C}$ with $5 \% \mathrm{CO}_{2}$ 
overnight. The next day, the media was replaced with 90\% McCoy's 5A medium. Five days later the cells were analyzed on a Gallios flow cytometer (Beckman Coulter, Brea, CA, USA) to ensure infection. The cells that were positive for IRFP702 were sorted from the negative population on a MoFlo Astrios EQ Cell Sorter (Beckman Coulter). IRFP702 expression in the cells was confirmed via scanning and imaging in the 700 channel on the Odyssey CLx (LI-COR Biosciences, Lincoln, Nebraska, USA). For fluorescence analyses, the fluorescent cells and matching non-fluorescent cells (control) were seeded on coverslips in 6-well culture dishes and cultured overnight in McCoy's 5A Media. The commercial glass slides (Cat. \# 12-552-3, Fisher Scientific, USA) and coverslips (Cat. \# 12-540C, Fisher Scientifc, USA) have standard dimensions of $75 \mathrm{~mm} \times 25 \mathrm{~mm}$ (length $\times$ width) and $25 \mathrm{~mm} \times 25 \mathrm{~mm}$ (length $\times$ width) respectively. An EVOS Cell Imaging System (Thermo Fisher Scientific) was used to follow the growth of the cells. The cells were then fixed using 1\% paraformaldehyde (PFA) (Sigma-Aldrich) on a glass slide which was used to test the prototype.

\section{Results and Discussion}

Figure 4a illustrates the experimental setup of our developed prototype, while Figure $4 \mathrm{~b}$ is an image of the commercial system (Li-Cor Odyssey) used to visualize the cancer cells with and without conjugating fluorophore. The resulting device is portable, which uses less power, costs less, is small in size as well as lightweight and provides an alternative to the conventional fluorescent microscope. The photodiode is kept inside the custom-built sample chamber and the flashlight is held on top of the cultured sample. When the light from the excitation source excites the cultured sample of HCT116 cells expressing IRFP702, a fluorescent signal is produced. At the receiving end, the light is collected by the photodiode and is converted to the current, which is linearly proportional to the collected light [24]. The photodiode is reverse biased with the help of Arduino Uno's $5 \mathrm{~V}$ pin, and the anode is connected to the ground via a resistor. The Arduino Uno has 5 analog pins (A0-A5). The photodiode is connected to the Arduino Uno through a A0 pin. When the light strikes the photodiode, it causes the current to flow through the resistor, which causes the voltage across it. The sensitivity of photodiode was adjusted by utilizing this principle.

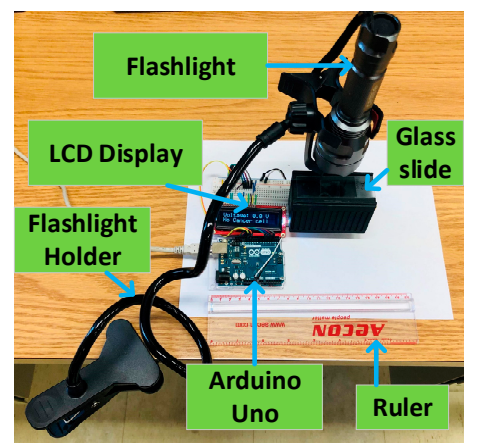

(a)

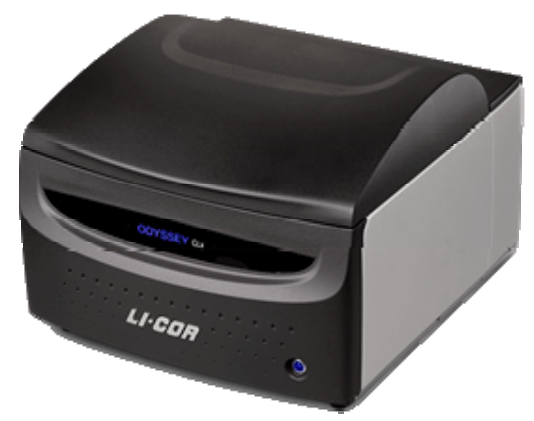

(b)

Figure 4. Experimental setup: (a) an overall setup of the proposed system (ruler is added in the picture to show the relative size) and (b) a commercial system: Li-Cor Odyssey.

In order to test the minimum confluency required for the proposed device to work successfully, we cultured the cells of different confluency on the coverslips. The confluency of the cells varied from $10 \%$ to $90 \%$ in 10 percent increments. The samples were distributed evenly throughout the coverslip. Four samples of each confluency were cultured on different glass slides in order to test the performance of the proposed device. A confluency of $100 \%$ was mapped to 0.3 million cells per coverslip. The other confluency level was set accordingly. It was concluded that a minimum of $60 \%$ confluency is required for the proposed device to work successfully. It is to be noted that the developed device was able to get readings from the samples that were less than $60 \%$ confluent. However, the obtained readings from these samples were not conclusive as the readings were variable. Since the readings were not 
consistent, the remaining experiments were performed by keeping the samples at a minimum of $60 \%$ confluent. Moreover, the distance between the sample and the flashlight was fixed at $2 \mathrm{~cm}$ with the help of a holder.

Figure 5a shows the cultured HCT116 cells expressing IRFP702, and Figure 5b is the control cells when viewed under a commercial imaging system (Odyssey CLx, LI-COR Biosciences, Lincoln, NE, USA). The same cell line that was used with the commercial system [25] was then used with our prototype to validate the reading. The proposed prototype was able to detect the emitted fluorescent signal from the HCT116 colorectal cancer cells expressing IRFP702 and hence was able to distinguish between control cells and the fluorescent cells. The excitation and emission maximum of IRFP702 fluorophore is $673 \mathrm{~nm}$ and $702 \mathrm{~nm}$ respectively, as shown in Figure 5d. The optical filter and light source as well as the photodiode were chosen cautiously so that they cover the excitation and emission region of this IRFP702 fluorophore. The longpass filter was selected so that it matches the emission wavelength of the IRFP702 fluorophore, thus ensuring that the emitted fluorescent wavelength passes down to the photodiode. This is to ensure that the light that is detected by the photodiode is from the cell line and not from the excitation source. Although a dichroic mirror/excitation filter is usually used in combination with the excitation light source $[7,26]$, this work shows that extra components can be removed if the experimental set-up is performed cautiously as it was also shown in [27].

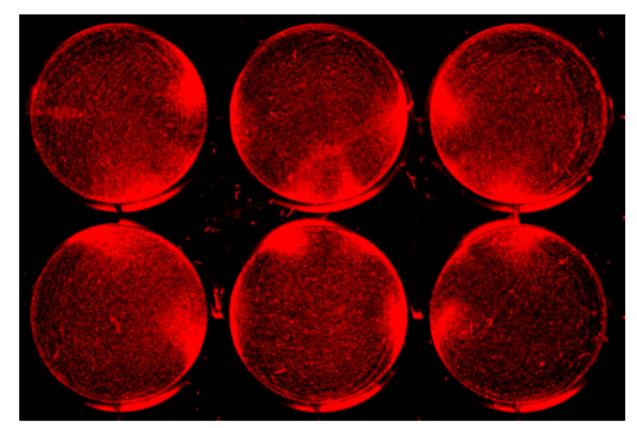

(a)

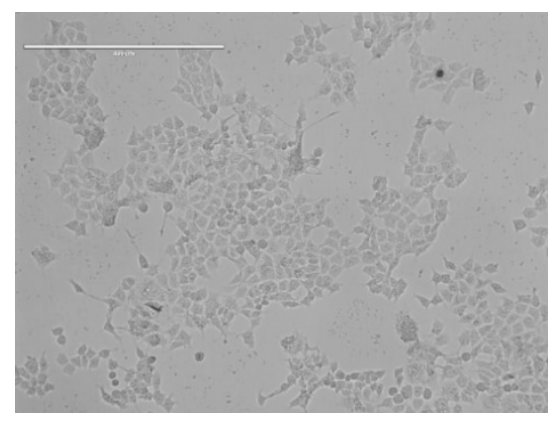

(c)

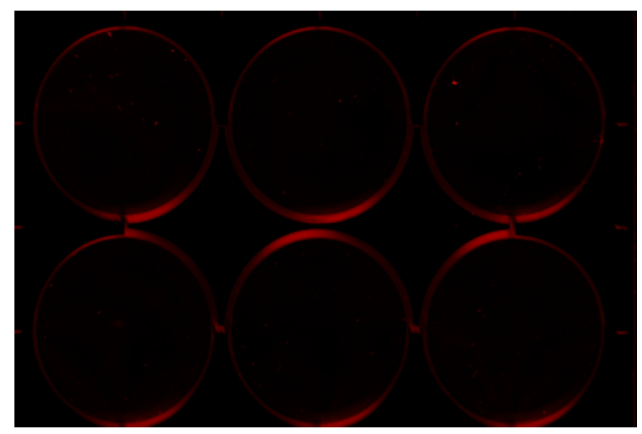

(b)

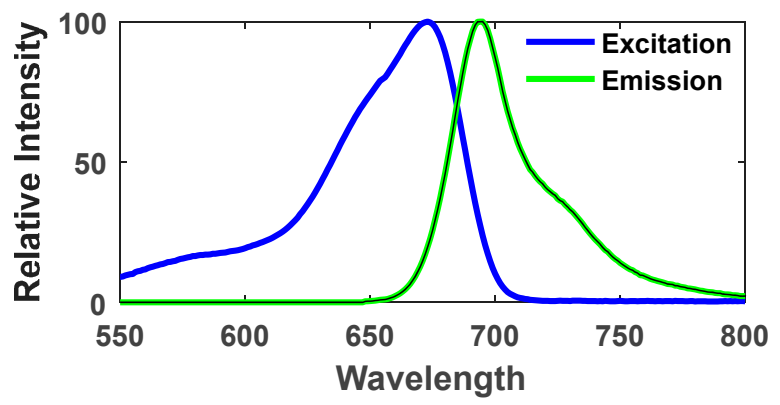

(d)

Figure 5. Detection of colorectal cancer cells expressing IRFP702: (a) colorectal cancer cells (HCT116) with IRFP702 fluorophore expression visualized under Odyssey CLx NIR scanner (LI-COR), (b) colorectal cancer cells (HCT116) without IRFP702 fluorophore expression visualized under Odyssey CLx NIR scanner (LI-COR), (c) colorectal cancer cells with IRFP702 fluorophore expression under EVOS Cell Imaging System, (d) excitation and emission spectra of IRFP702. This figure shows the absorption and emission spectra of the IRFP702 where the peak absorption and peak emission of the cultured sample is $673 \mathrm{~nm}$ and $702 \mathrm{~nm}$ respectively.

The complete system is powered and controlled by the help of a microcontroller (Arduino Uno). It detects the signal and transmits the measured signal to the LCD display. This microcontroller converts the analog signal generated by the photodiode using a high-precision 10-bit analog to digital converter (ADC) which is embedded inside the microcontroller. The photodiode is a semiconductor 
device which converts light into current [28]. The current is produced when the photons are taken up by the photodiode. In Arduino Uno's ADC, the analog signal, which ranges from 0 to $5 \mathrm{~V}$, is translated into a 10-bit code (1024 combinations) which corresponds to the analog value ranging from 0 to 1023 . The Arduino Uno can read a maximum input voltage of $5 \mathrm{~V}$, i.e., any voltage that is higher than $5 \mathrm{~V}$ will be read as $5 \mathrm{~V}$ regardless of their actual values. To remove this limitation, resistors of different values can be used to limit the voltage within $5 \mathrm{~V}$. However, in this work, the obtained readings did not go beyond the maximum limit of the Arduino Uno as the generated fluorescence signal is not very strong. Figure 6 shows a flowchart which summarizes the working of the developed prototype. An Arduino IDE compiler is used to program the microcontroller. The code is written in C language. The device is initialized when it is first powered on. After the initial boot is successful, a sample of a control cell, which is cultured on a glass slide, is placed in the sample holder. The sample holder has a port which is designed to fit the cultured samples in it. After the sample placement is successful, the device starts reading. The microcontroller reads the data 10 times in order to reduce errors that may be caused due to manipulation, movement or placement of flashlight. The measured value is averaged, which is saved as a base value and displayed on the LCD display. This value will be used later in the calculation. It is to be noted that it is important to place the excitation source/flashlight on top of the sample, which is why a flashlight holder is used. The holder helps the user to hold the flashlight on top of the sample at the desired angle and distance away from the sample. After the reading of the control cell is completed, it is replaced with a sample of fluorescent colorectal cancer cell (HCT116 conjugated with IRFP702). As in the earlier case, the reading is performed again 10 times. This reading is averaged and compared with the previously measured value from the control cell. If the difference in voltage measurement obtained from the control cell and the fluorescent cell is greater than $700 \mathrm{mV}$, "CancerCell Found" is displayed. Otherwise, "No CancerCell" is displayed.

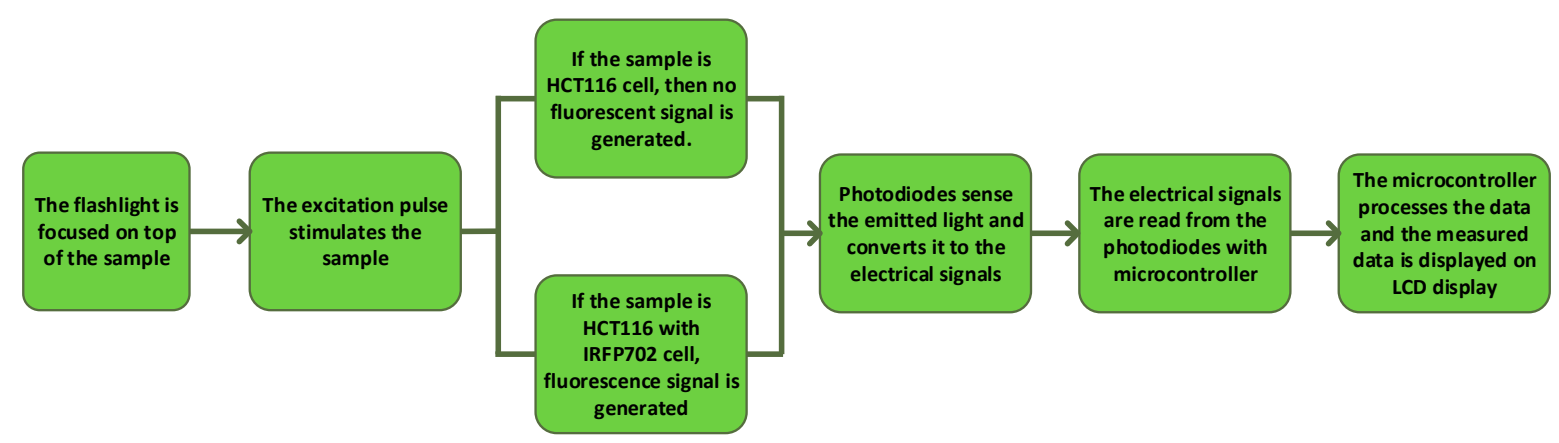

Figure 6. Flowchart showing the operating procedure of the device.

Figure 7a,b show the control colorectal cancer cells and the IRFP702 expressing colorectal cancer cells on a glass slide respectively. Figure $7 \mathrm{c}$,d show the control cells and the IRFP702 expressing colorectal cancer cells visualized with the help of a commercial system [25] respectively. From Figure $7 \mathrm{a}, \mathrm{b}$, it can be construed that it is difficult to differentiate between these cultured samples with the naked eye. However, when these same cultured colorectal cancer cells are used with our developed device, we are able to differentiate between these cells, as can be seen in Figure 7e. These readings from the samples were visualized with the help of waveform oscilloscope on a computer. In order to evaluate the effectiveness/performance of the proposed device, we applied four measurement techniques that are commonly used in classification. These measurements include sensitivity, accuracy, specificity and precision, where sensitivity $=\mathrm{TP} /(\mathrm{TP}+\mathrm{FN})$, accuracy $=(\mathrm{TP}+\mathrm{TN}) /(\mathrm{TP}+\mathrm{TN}+\mathrm{FP}+\mathrm{FN})$, specificity $=\mathrm{TN} /(\mathrm{TN}+\mathrm{FP})$ and precision $=\mathrm{TP} /(\mathrm{TP}+\mathrm{FP})$. As shown in Figure $8 \mathrm{a}$, the fluorescence intensity is proportional to the number of cells. 


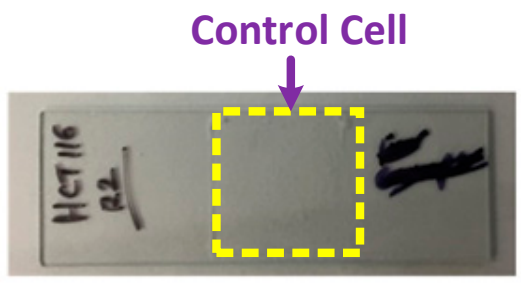

(a)

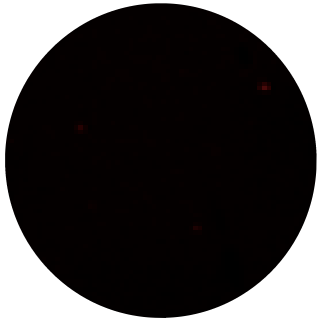

(c)

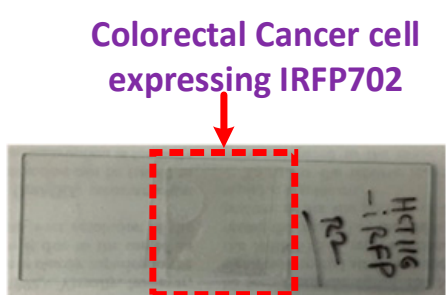

(b)

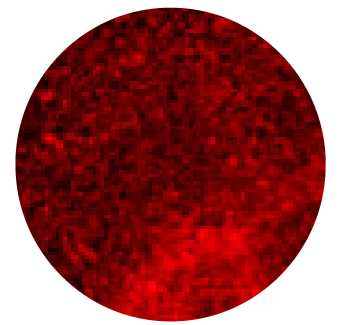

(d)

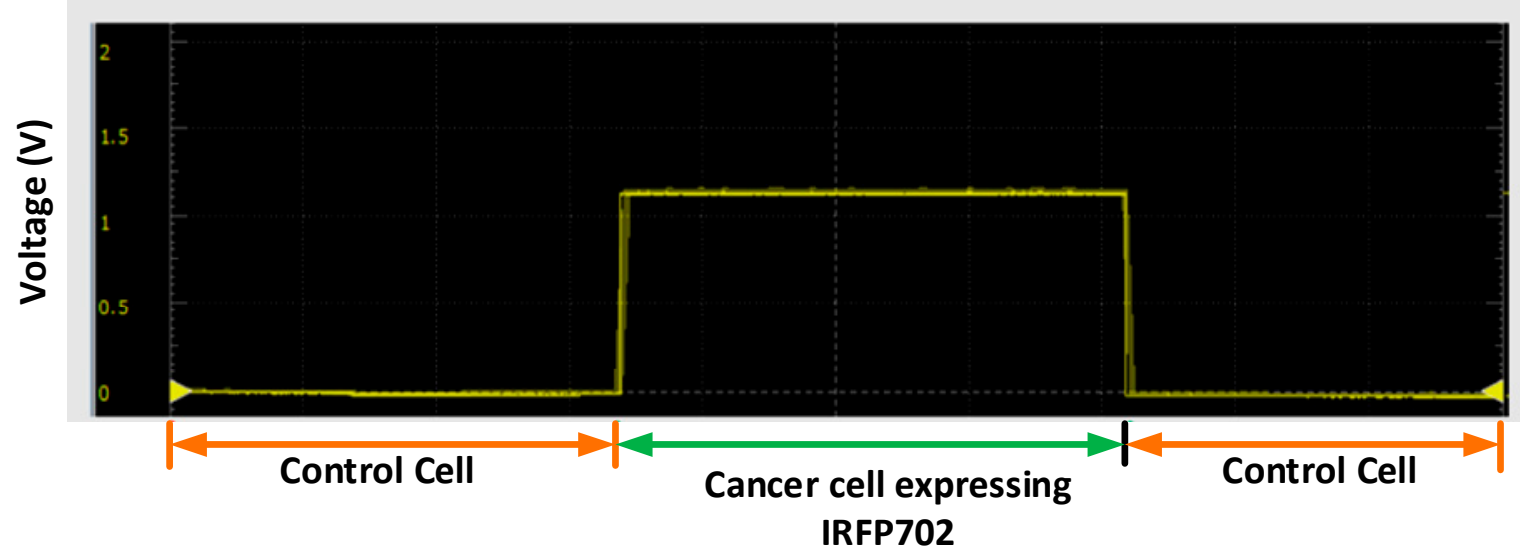

(e)

Figure 7. Results: (a) control colorectal cancer cell, (b) colorectal cancer cell expressing IRFP702 fluorophore, (c) control cell visualized with commercial system, (d) colorectal cancer cell expressing IRFP702 visualized with commercial system, and (e) reading on a waveform oscilloscope.

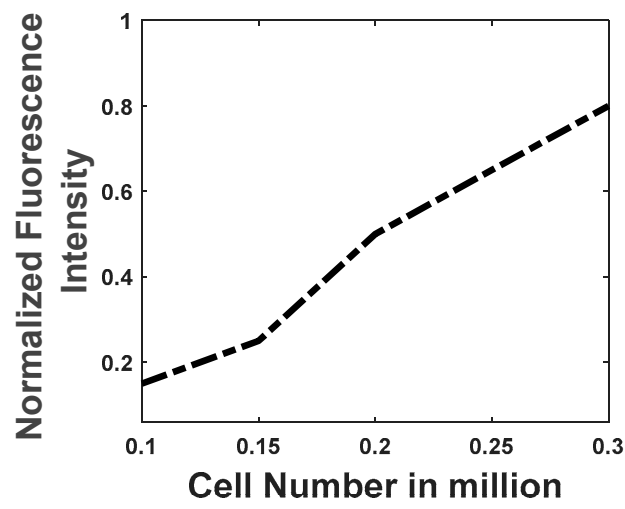

(a)

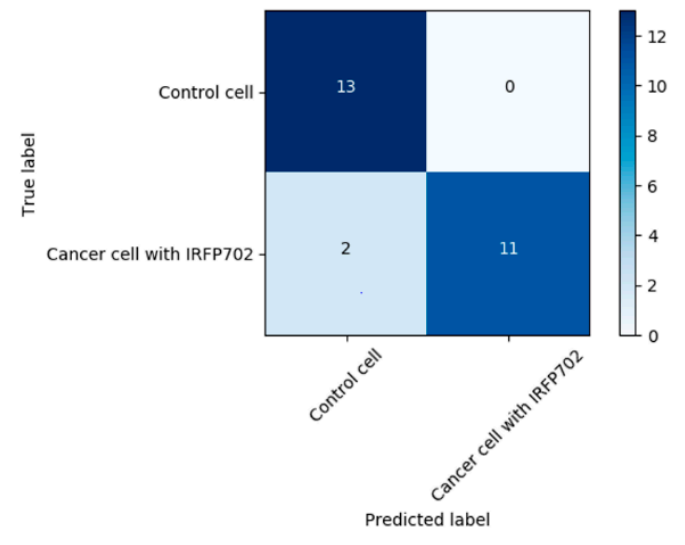

(b)

Figure 8. (a) Fluorescence intensity vs cell-number (b) Confusion matrix

Twenty-six samples (thirteen of cancer cell expressing IRFP702 and thirteen of control cell) were cultured on the glass slide with a confluency greater than $60 \%$, and they were examined first with the Odyssey system and then with the developed prototype. Figure $8 \mathrm{~b}$ shows the confusion matrix. As can 
be seen in Figure $8 b$, the proposed device was able to detect all of the control cells from the samples accurately. On the other hand, the fluorimeter detected 11 out of 13 cancer cells with IRFP702 correctly. The sensitivity, accuracy, specificity and precision were found out to be $0.85,0.92,1$ and 1 respectively.

Table 1 shows the list of major components that were used to build this device along with the individual and aggregate cost. The total cost of this device can further be reduced by using a low-cost filter which can provide similar accuracy. The presented device is again simplified with respect to a regular fluorescence microscope [7] as it eliminates the requirement of an additional dichroic mirror as well as excitation filter. Moreover, it is small in size, compact, costs less and is lightweight when compared to most commercial as well as research microscopes, as shown in Table 2. In addition to these advantages, removing the filter and camera also offers additional advantages, such as it eliminates the need of having to adjust light path settings and the external arc lamp. This device also obviates the need of having a filter cube in these devices to house the different set of filters. In addition, this device also removes the need for using expensive cameras. The sample chamber takes up a space of $4 \times 8 \mathrm{~cm}$ which makes this experimental setup a perfect candidate for benchtop experiments. This device is user-friendly as the instruction is displayed on the LCD screen guiding the user for each step in the detection process. Alternatively, this developed device can also be connected via the serial port of a computer with a USB to enable control by the computer and hence control input and output operations via the computer through serial communication. We first tested the functionality of the developed fluorimeter by using it to detect fluorescence emitted from human colorectal cancer cells which were genetically engineered to express the fluorescence protein. These cultured cells functioned both as a biologically appropriate as well as technically convenient clinical proxy of colorectal cancer cells for the fluorescence-based selective-detection process. As proof of principle, the cultured colorectal cancer cells (with and without IRFP702) were imaged using a conventional Odyssey CLx imaging system obtained commercially from Odyssey and the same cultured cells (with and without IRFP702) were then tested with our developed prototype to validate the result.

Table 1. List of major components required to construct the proposed device.

\begin{tabular}{ccc}
\hline Components Used & Model/Specifications & Estimated Cost (USD) \\
\hline Filter [29] & $700 \mathrm{~nm}$ & 150 \\
3D printed chamber & Printed with CR-10 & 30 \\
16 2 LCD display [22] & HC1624, 5 V, 16 $\times 2$ & 3.90 \\
Breadboard with wires [30] & Microtivity & 6.09 \\
Microcontroller [21] & Arduino Uno R3 & 16.90 \\
Flashlight [18] & UltraFire C8 & 6.58 \\
Photodiode [19] & Mouser, 720-SFH203 & 0.94 \\
Flashlight holder [31] & Any cell phone holder with clip & 10 \\
& Total & $\mathbf{2 2 4 . 3 7}$ \\
\hline
\end{tabular}


Table 2. Comparison of the developed device with other devices in market or in research.

\begin{tabular}{|c|c|c|c|c|c|c|c|}
\hline & Work. & Weight (kg) & Dimension (cm) & $\begin{array}{l}\text { Cost } \\
\text { (USD) }\end{array}$ & Target of Interest & $\begin{array}{c}\text { Standalone/Needs pc } \\
\text { with Software }\end{array}$ & Power Source \\
\hline \multirow{5}{*}{ Image Based } & Tapley et al. (2013) [3] & 3 & $20 \times 20 \times 10$ & Not known & Tuberculosis & Needs PC & Battery Powered \\
\hline & Nunez et al. (2017) [32] & Not known & $17 \times 18.7 \times 31$ & $\$ 250$ & Imaging Assays & Needs PC for analysis & AC powered \\
\hline & Hasan et al. (2016) [7] & 0.13 & $11 \times 6.5 \times 15$ & $\$ 358$ & Breast Cancer & $\begin{array}{l}\text { Needs PC for } \\
\text { visualization }\end{array}$ & Battery Powered \\
\hline & Babbit et al. (2013) [33] & Not known & Not known & $\$ 772.93^{*}$ & Living cells & Standalone & Battery Powered \\
\hline & $\begin{array}{c}\text { Commercial microscope } \\
\text { (2019) [25] }\end{array}$ & 33 & $93.98 \times 134.62 \times 157.48$ & $\begin{array}{c}\$ 50,055.42 \\
{[34]}\end{array}$ & Multiple & Needs PC & $\mathrm{AC}$ powered \\
\hline \multirow{7}{*}{ Signal Based } & Lamb et al. (2015) [35] & Not known & $4 \times 4^{* *}$ & $\$ 3300$ & Chlorophyll & $\begin{array}{c}\text { Needs auxiliary } \\
\text { component }\end{array}$ & AC powered \\
\hline & Hoadley et al. (2017) [36] & Not known & Not known & $\$ 712.44$ & Chlorophyll & $\begin{array}{l}\text { Standalone / needs pc } \\
\text { for post-processing }\end{array}$ & AC powered \\
\hline & Blockeistein et al. (2014) [37] & Not known & $7.6 \times 7.6 \times 12.7$ & $<\$ 500$ & Chlorophyll & $\begin{array}{l}\text { Needs a pc with } \\
\text { software }\end{array}$ & AC powered \\
\hline & $\begin{array}{l}\text { Commercial fluorometer } \\
\text { (2019) [38] }\end{array}$ & 55 & $104 \times 59 \times 32$ & Not known & Multiple & Needs pc with software & AC powered \\
\hline & Martin et al. (2016) [39] & Not known & $22 \times 100 \times 5.3$ & $>\$ 170 *$ & Escherichia & Standalone & $\begin{array}{l}\text { AC / Battery } \\
\text { powered }\end{array}$ \\
\hline & Kim et al. *** (2017) [11] & 0.108 & $5.2 \times 3.5 \times 2.5$ & Not known & Cyanobacteria & Standalone & 9 V supply \\
\hline & Proposed Device & 0.16 & $8 \times 4 \times 3.2^{\dagger}$ & $\$ 224.37$ & Colorectal Cancer & Standalone & $\begin{array}{l}\text { AC / Battery } \\
\text { powered }\end{array}$ \\
\hline
\end{tabular}

${ }^{*}$ Not reported in detail. The total cost was calculated based on the list of equipment provided in the manuscript; ${ }^{* *}$ height is not mentioned in the manuscript; ${ }^{* * *}$ weight and dimension is without considering laser source or sample vial; ${ }^{\dagger}$ Size of sample chamber. 


\section{Conclusions}

In this paper, we proposed an alternative low-cost fluorimeter which can differentiate between cultured control cell and the fluorescent colorectal cancer cell. The choice of the light source, optical filter and the sensor is critical in the successful implementation of this device. The device can be minimized further to make it more portable, appealing and lightweight. The Arduino Uno used in this project contains an Atmega chip, which can be programmed and the unnecessary component or pins can be removed while keeping only the necessary components that are required for this device [40]. In addition to this, the flashlight can be replaced with another light source which works in the same range but is lightweight and portable. Following this process will make this device smaller as well as portable. Alternatively, the Arduino Uno can be replaced by other boards which are relatively smaller in size [41]. This research aims to construct a fluorimeter and make it accessible to medical and research laboratories where resources are limited. This goal is realized as we demonstrate the functionality of this device using low-cost, easily available off-the-shelf components. Hence, the manufacturing of this device is possible by a wide range of laboratories across the globe. This device can be used to detect other types of cancer cells by simply replacing the optical filter and detector circuitry, which must be selected according to the conjugated or expressed fluorophores [12]. Moreover, this device can also be tested to detect other targets in the future with slight modifications, such as chlorophyll, Escherichia and cyanobacteria. The lightweight, easy-to-use, portable and compact design of this device, as well as the potential for measurement of multiple types of cancer in the near future, will foster research in many laboratories around the globe without the financial burden of the cumbersome commercial instrument.

Author Contributions: Conceptualization, M.W.A.; methodology, M.W.A.; software, M.W.A.; validation, M.W.A., M.F.I. and W.B.; formal analysis, M.W.A.; resources, K.A.W., C.R.G. and F.J.V.; writing—original draft preparation, M.W.A.; writing — review and editing, M.W.A., M.F.I., W.B. and K.A.W.; visualization, M.W.A., K.A.W. and M.F.I.; supervision, K.A.W., C.R.G. and F.J.V.; funding acquisition, K.A.W.

Funding: This research was funded by the Natural Sciences and Engineering Research Council of Canada.

Acknowledgments: The authors thank Tanin Sultana for her suggestions in this work.

Conflicts of Interest: The authors declare no conflicts of interest.

\section{References}

1. Moerner, W.E.; Fromm, D.P. Methods of single-molecule fluorescence spectroscopy and microscopy. Rev. Sci. Instrum. 2003, 74, 3597-3619. [CrossRef]

2. Cole, D.; Young, G.; Weigel, A.; Sebesta, A.; Kukura, P. Label-Free Single-Molecule Imaging with Numerical-Aperture-Shaped Interferometric Scattering Microscopy. ACS Photonics 2017, 4, 211-216. [CrossRef] [PubMed]

3. Tapley, A.; Switz, N.; Reber, C.; Davis, J.L.; Miller, C.; Matovu, J.B.; Worodria, W.; Huang, L.; Fletcher, D.A.; Cattamanchi, C. Mobile Digital Fluorescence Microscopy for Diagnosis of Tuberculosis. J. Clin. Microbiol. 2013, 51, 1774-1778. [CrossRef] [PubMed]

4. Kim, B.; Lee, Y.J.; Park, J.G.; Yoo, D.; Hahn, Y.K.; Choi, S. A portable somatic cell counter based on a multi-functional counting chamber and a miniaturized fluorescence microscope. Talanta 2017, 170, 238-243. [CrossRef] [PubMed]

5. Akraa, S.; Tam AP, T.; Shen, H.; Tang, Y.; Tang, B.Z.; Li, J.; Walker, S. A smartphone-based point-of-care quantitative urinalysis device for chronic kidney disease patients. J. Netw. Comput. Appl. 2018, 115, 59-69. [CrossRef]

6. Kissinger, J.; Wilson, D. Portable Fluorescence Lifetime Detection for Chlorophyll Analysis in Marine Environments. IEEE Sens. J. 2011, 11, 288-295. [CrossRef]

7. Hasan, M.M.; Alam, M.W.; Wahid, K.A.; Miah, S.; Lukong, K.E. A Low-Cost digital microscope with real-Time fluorescent imaging capability. PLoS ONE 2016, 11, e0167863. [CrossRef] [PubMed] 
8. Zheng, Y.; Zhang, L.; Mi, T.; Zhao, J. Portable plant chlorophyll fluorimeter based on blue LED rapid induced technology. In Proceedings of the 2017 International Conference on Optical Instruments and Technology: Optoelectronic Measurement Technology and Systems, Beijing, China, 28-30 October 2017; p. 45.

9. Geng, X.; Gao, Y.; Feng, C.; Guan, Y. A facile and high sensitive micro fluorimeter based on light emitting diode and photodiode. Talanta 2017, 175, 183-188. [CrossRef] [PubMed]

10. Xing, X.; Claustre, H.; Boss, E.; Roesler, C.; Organelli, E.; Poteau, A.; Barbieux, M.; D'Ortenzio, F. Correction of profiles of in-situ chlorophyll fluorometry for the contribution of fluorescence originating from non-algal matter. Limnol. Oceanogr. Methods 2017, 15, 80-93. [CrossRef]

11. Kim, S.H.; He, Y.; Lee, E.H.; Kim, J.H.; Park, S.M. Portable Fluorometer for Cyanobacteria Detection. IEEE Sens. J. 2017, 17, 2377-2384. [CrossRef]

12. Alam, M.W.; Wahid, K.A.; Goel, R.K.; Lukong, K.E. Development of a low-cost and portable smart fluorometer for detecting breast cancer cells. Biomed. Opt. Express 2019, 10, 399-410. [CrossRef] [PubMed]

13. Siegel, R.L.; Miller, K.D.; Jemal, A. Cancer statistics, 2017. CA Cancer J. Clin. 2017, 67, 7-30. [CrossRef] [PubMed]

14. Estrada, J.J.; Bedon, A.M.; Kaminski, J.P.; Rodriguez, G.; Ali, M.; Albert, A. Direct access screening colonoscopy as a safe and effective approach to increasing colorectal cancer screening. J. Clin. Oncol. 2017, 35, 540. [CrossRef]

15. Gautier, A.; Tebo, A.G. Fluorogenic Protein-Based Strategies for Detection, Actuation, and Sensing. BioEssays 2018, 40, 1800118. [CrossRef] [PubMed]

16. Zhang, R.R.; Schroeder, A.B.; Grudzinski, J.J.; Rosenthal, E.L.; Warram, J.M.; Pinchuk, A.N.; Weichert, J.P. Beyond the margins: Real-time detection of cancer using targeted fluorophores. Nat. Rev. Clin. Oncol. 2017, 14, 347-364. [CrossRef] [PubMed]

17. McCann, T.E.; Kosaka, N.; Choyke, P.L.; Kobayashi, H. The Use of Fluorescent Proteins for Developing Cancer-Specific Target Imaging Probes; Humana Press: Totowa, NJ, USA, 2012; pp. 191-204.

18. UltraFire CG-C8 Flashlight. Available online: https://www.amazon.co.uk/UltraFire-CG-C8-3-ModeFlashlight-1x18650/dp/B00JA9EMLK (accessed on 25 February 2019).

19. SFH 203 OSRAM Opto Semiconductors | Mouser. Available online: https://www.mouser.com/ProductDetail/OSRAM-Opto-Semiconductors/SFH-203?qs= sGAEpiMZZMtWNtIk7yMEsZEKXNTNxzvbCuJXkQkukYk\%3D (accessed on 25 February 2019).

20. SFH 203 Photodiode. Available online: https:/media.osram.info/media/resource/hires/osram-dam-2495935/ SFH203.pdf (accessed on 25 February 2019).

21. Arduino Uno Rev3 SMD. Available online: https://store.arduino.cc/usa/arduino-uno-smd-rev3 (accessed on 25 February 2019).

22. Basic 16x2 Character LCD—LCD-09052, Sparkfun. Available online: https://www.sparkfun.com/products/ 9052 (accessed on 25 February 2019).

23. Cree. Product Family Data Sheet Cree®XLamp®XP-E LEDs. 2013. Available online: http://www.cree.com/ led-components/media/documents/XLampXPE.pdf (accessed on 5 December 2018).

24. Alam, M.W.; Sultana, T.; Alam, M.S. A heartbeat and temperature measuring system for remote health monitoring using wireless body area network. Int. J. Bio Sci. Bio Technol. 2016, 8, 171-190. [CrossRef]

25. Odyssey. Available online: https://www.licor.com/bio/products/imaging_systems/odyssey/ (accessed on 19 November 2018).

26. Witte, R.; Andriasyan, V.; Georgi, F.; Yakimovich, A.; Greber, U. Concepts in Light Microscopy of Viruses. Viruses 2018, 10, 202. [CrossRef] [PubMed]

27. Lamb, J.J.; Eaton-Rye, J.J.; Hohmann-Marriott, M.F. A Cost-Effective Solution for the Reliable Determination of Cell Numbers of Microorganisms in Liquid Culture. Curr. Microbiol. 2013, 67, 123-129. [CrossRef] [PubMed]

28. Das, C.K.; Alam, M.W.; Hoque, I. A Wireless heartbeat and Temperature Monitoring System for Remote Patients. In Proceedings of the International Conference on Mechanical Engineering and Renewable Energy 2013 (ICMERE2013), Chittagong, Bangladesh, 1-3 May 2014; pp. 1-6.

29. 700nm High Performance Longpass Filter, Edmund Optics. Available online: https://www.edmundoptics. com/p/700nm-125mm-dia-high-performance-longpass-filter/17597/ (accessed on 25 February 2019).

30. Microtivity IB401. Available online: https://www.amazon.com/microtivity-400-point-ExperimentBreadboard-Jumper/dp/B004RXKWDQ (accessed on 25 February 2019). 
31. Cell Phone Holder. Available online: https://www.amazon.com/dp/B01H8RDAX6 (accessed on 3 March 2019).

32. Nuñez, I.; Matute, T.; Herrera, R.; Keymer, J.; Marzullo, T.; Rudge, T.; Federici, F. Low cost and open source multi-fluorescence imaging system for teaching and research in biology and bioengineering. PLOS ONE 2017, 12, e0187163. [CrossRef]

33. Babbit, K.N.; Hanzlik, G.A.; Busse, C.A. Observing fluorescent probes living cells using a low-cost LED flashlight retrofitted to a common vintage light microscope. J. Microbiol. Biol. Educ. 2013, 1, 121-124. [CrossRef]

34. General Services Administration, Federal Supply Service. 2012. Available online: https://www.gsaadvantage. gov/ref_text/GS24F1183C/0LJVOV.2LOISL_GS-24F-1183C_TERMSCONDITIONS1183C.PDF (accessed on 19 November 2018).

35. Lamb, J.; Forfang, K.; Hohmann-Marriott, M. A Practical Solution for 77 K Fluorescence Measurements Based on LED Excitation and CCD Array Detector. PLoS ONE 2015, 10, e0132258. [CrossRef] [PubMed]

36. Hoadley, K.D.; Warner, M.E. Use of Open Source Hardware and Software Platforms to Quantify Spectrally Dependent Differences in Photochemical Efficiency and Functional Absorption Cross Section within the Dinoflagellate Symbiodinium spp. Front. Mar. Sci. 2017, 4, 365. [CrossRef]

37. Blockstein, L.; Yadid-Pecht, O. Lensless Miniature Portable Fluorometer for Measurement of Chlorophyll and CDOM in Water Using Fluorescence Contact Imaging. IEEE Photonics J. 2014, 6, 1-16.

38. FS5 Spectrofluorometer | Steady State | Edinburgh Instruments. Available online: https://www.edinst.com/ us/products/fs5-spectrofluorometer/ (accessed on 8 November 2018).

39. Martín, F.J.F.; Llopis, M.V.; Rodriguez, J.C.C.; Fernández, L.M.; Menéndez, I.G.D.R.; Fernández, J.F.; Brugos, F.L.; Fernandez, N.C.; Corugedo, F.O.F.; Suarez, I.M. A Novel Handheld Fluorimeter for Rapid Detection of Escherichia coli in Drinking Water. IEEE Sens. J. 2016, 16, 5136-5144. [CrossRef]

40. How to Make a Permanent Circuit Board to Shrink Arduino Projects | Arduino | Maker Pro. Available online: https://maker.pro/arduino/tutorial/how-to-make-a-permanent-circuit-board-to-shrink-arduinoprojects (accessed on 1 August 2019).

41. PICO: The world's smallest Arduino compatible board! by MellBell Electronics—Kickstarter. Available online: https://www.kickstarter.com/projects/melbel/pico-the-worlds-smallest-arduino-board/faqs (accessed on 27 July 2019).

(C) 2019 by the authors. Licensee MDPI, Basel, Switzerland. This article is an open access article distributed under the terms and conditions of the Creative Commons Attribution (CC BY) license (http://creativecommons.org/licenses/by/4.0/). 